\title{
Theoretical and Empirical Developments of the Mindfulness-Acceptance-Commitment (MAC) Approach to Performance Enhancement
}

\author{
Zella E. Moore \\ Manhattan College
}

\begin{abstract}
As long as athletes strive to attain optimal performance states and consistently reach high performance goals, psychological interventions will be used to assist in the development of skill and the maintenance of performance. In the pursuit of these goals, newer evidence-driven models based on mindfulness- and acceptance-based approaches have been designed to achieve these ends. Based upon questionable efficacy data for traditional psychological skills training procedures that emphasize reduction or control of internal processes, mindfulness- and acceptance-based approaches develop skills of nonjudging mindful awareness, mindful attention, and experiential acceptance to aid in the pursuit of valued goals. The most formalized and researched mindfulness- and acceptance-based approach within sport psychology is the manualized Mindfulness-Acceptance-Commitment (MAC) protocol. In the 8 years since the MAC was first developed and presented, and the 5 years since the first publication on the protocol, the MAC program has accumulated a continually growing empirical base for both its underlying theory and intervention efficacy as a performance enhancement intervention. This article reviews the empirical and theoretical foundations of the mindfulness- and acceptance-based approaches in general, and MAC in particular; reviews the accumulated empirical findings in support of the MAC approach for performance enhancement; and presents recent MAC developments and suggested future directions.
\end{abstract}

It has been nearly a decade since the first professional conference presentation introducing the Mindfulness-Acceptance-Commitment (MAC) approach to performance enhancement (Moore \& Gardner, 2001), a theoretically and empirically derived acceptance-based behavioral intervention for the purpose of enhancing the performance and psychological well-being of competitive athletes. Since that time, many advances have occurred in the field of study broadly referred to as acceptance-based behavior therapies (ABBT; Roemer \& Orsillo, 2009). This term is used to refer to a broad range of interventions that view the alteration of

The author is with the Department of Psychology at Manhattan College in New York City. 
an individual's relationship to, and avoidance of, internal experiences such as thoughts and emotions, as a primary mechanism of change. An integral subset of acceptance-based approaches is mindfulness, which has been defined as an "openhearted, moment-to-moment non-judgmental awareness" (Kabat-Zinn, 2005, p. 24). The primary purpose of this paper is to summarize the theoretical development of ABBT in general (including its mindfulness components) and MAC in particular, note relevant empirical studies that have been published since the inception of MAC that support and enhance its basic foundation, and provide a picture of how it has evolved over time as a relevant and clear intervention alternative to the traditional change-based psychological skills training (PST) approaches common to sport psychology research and practice.

The Mindfulness-Acceptance-Commitment (MAC) program's development was initially borne out of the recognition that empirical support for the efficacy of traditional psychological skills training procedures was lacking (Gardner \& Moore, 2007; Moore, 2003; Moore \& Gardner, 2001) and drew upon the theoretical developments emerging from the "third-wave" acceptance-based behavioral movement in clinical psychology (Hayes, Strosahl, \& Wilson, 1999). Of course, the criticism of a lack of commitment to an evidence-based approach to practice is not unique to sport psychology. In fact, recent critiques have suggested that many practitioners of clinical psychology fail to remain committed to the evidence-based professional model of practice and instead rely on subjective beliefs and/or experience in making intervention choices (Baker, McFall, \& Shoham, 2009); however, sport psychology has been particularly challenged to even develop a sufficient base for its professional practice. Thus, as a result of what we (the developers of the MAC approach) saw as a unacceptable state of affairs with regard to the lack of evidence base in applied sport psychology, and due to rapid advances in both theoretical and applied studies of mindfulness- and acceptance-based interventions within mainstream psychology, the MAC was a theoretically conceived alternative approach to understanding athletic performance and working with athletes for the purpose of enhancing sport performance and overall well-being (Gardner, 2001; Gardner \& Moore, 2004; Moore \& Gardner, 2001, 2002, 2003).

Foundational to traditional PST approaches to performance enhancement is the idea that optimal performance is most likely to occur as athletes develop the capacity to control internal states such as cognitions, emotions, and sensations, in order to achieve a "necessary" internal state (Hardy, Jones, \& Gould, 1996) believed to support high level athletic performance. The intervention goal of achieving control of internal experiences to create this optimal state of readiness for athletic performance is typified by traditional cognitive-behaviorally oriented PST techniques such as arousal control interventions, goal setting, imagery training, and self-talk interventions. In contrast to these "control" based interventions, which view the attainment of an optimal internal state as necessary for peak athletic performance, the acceptance-based foundation of MAC suggests an essentially opposite perspective. That is, optimal performance does not require the reduction or control of internal states but rather, requires a nonjudging (i.e., not good, not bad) moment-to-moment awareness and acceptance of one's internal state, whatever that may be, and an attentional focus on task-relevant external stimuli and behavioral choices that support one's athletic endeavor. 
In fact, perusal of the empirical literature within sport psychology clearly indicates that not only have traditional PST interventions not received sufficient efficacy standing despite 30 years of research, but possibly of greater importance, studies evaluating the mechanisms by which optimal performance actually occurs suggest a very different finding than would be predicted by the theoretical model from which PST interventions have been devised (see Gardner \& Moore, 2004, 2006 for a comprehensive review). In fact, many studies utilizing traditional PST procedures successfully reduced supposed "negative" internal experiences such as thoughts and emotions, and increased "positive" states such as confidence, yet demonstrated no significant increases in athletic performance despite these changes in internal states (e.g., Burton, 1989; Daw \& Burton, 1994; Maynard, Smith, \& Warwick-Evans, 1995; Murphy \& Woolfolk, 1987; Weinberg, Seabourne, \& Jackson, 1981). Findings from such studies suggest that while PST approaches may actually be effective in modifying those variables theorized to be related to athletic performance, no significant changes in athletic performance were found. The obvious conclusion from numerous studies reporting these same results is that reduction or control of these assumed mechanisms of action may not be relevant for optimal performance, as theorized.

Based on the paucity of outcome studies supporting the use of PST and the data-driven suggestion that the theoretical model and associated assumptions at the foundation of PST procedures lack empirical support, in 2001, Gardner and Moore (Gardner, 2001; Gardner \& Moore, 2004; Moore \& Gardner, 2001, 2002, 2003; Wolanin, Gardner, \& Moore, 2003) proposed the idea that rather than engaging in efforts to reduce, limit, or otherwise control internal experiences such as cognitions, emotions, and bodily sensations to create the elusive optimal performance state, prior research actually suggests that athletes can, and do, perform well with varying levels of internal experiences (Hanin, 1980). In fact, evidence suggests that efforts to "control" these naturally occurring internal states appear to be at best ineffective and at worst counterproductive (Gardner \& Moore, 2004; Sbrocco \& Barlow, 1996; Wegner, Ansfield, \& Pilloff, 1998; Wegner, \& Zanakos, 1994).

From this theoretical perspective, and in consideration of advances in mindfulness- and acceptance-based models within clinical psychology (Hayes, Strosahl, \& Wilson, 1999), Gardner and Moore (2004, 2006, 2007) took a completely different approach to understanding athletic performance, and intervention strategies and techniques designed to enhance athletic performance. From this convergence of prior disappointing empirical findings in sport psychology, and developing empirical findings within clinical psychology, the MAC took shape. Since its inception in 2001, a number of advances in the basic science of mindfulness- and acceptance-based interventions have occurred that have direct relevance for the further development and utilization of the MAC program.

\section{Recent Empirical Advances}

Empirical advances will be discussed in terms of recent developments in (a) basic science of acceptance-based models, (b) intervention studies within clinical/counseling psychology, and (c) intervention studies within sport psychology. 


\section{Developments Within the Basic Science of Acceptance- Based Models}

The most significant basic scientific studies relating to the constructs of mindfulness have come from studies designed to examine the effects that enhanced mindfulness and experiential acceptance have on physiological systems and executive (cognitive) functions. For example, empirical findings have clearly indicated that regular mindfulness practice and its associated enhanced awareness and attention result in increased immune functioning (Davidson et al., 2003) and enhanced alertness and improved orienting of attention (Jha, Krompinger, \& Baime, 2007). In an effort to provide a rationale for the connection of these findings to competitive sport, Marks (2008) suggested that through repeated exercise of attentional control and experiential acceptance by way of regular and consistent meditative practice, the athlete may automate a process of detecting and directing his or her attention to a desired stimulus without neglecting other relevant external information from the senses or internal information from within the body (e.g., autonomic arousal). By way of empirical support for this viewpoint, Chambers and colleagues (Chambers, Chuen Yee Lo, \& Allen, 2008) have demonstrated that increased mindfulness enhances sustained attention and attention switching, which they defined as the ability to switch one's attentional focus between relevant stimuli. Additional data in support of Marks' hypothesis comes from Brefczynski-Lewis and colleagues (2007), who, when studying experienced vs. less experienced meditators, found that the automated attentional process found in more experienced meditators may allow for more efficient allocation of cognitive resources. As such, enhanced levels of attentional focus can occur with lower levels of overall activation (i.e., overt effort). The implication of these findings is that by directing attentional resources more consistently, and with less possibility of engaging attention to surrounding cognitive "noise," such as random cognitions and emotions, the elite athlete maintains full or even heightened awareness of his or her context (i.e., the environmental cues inherent in the competitive sport), while simultaneously engaging in sustained on-task attention. Based on the empirical findings in studies on the relationship between mindfulness practice and neural correlates, it is reasonable also to assume (see Marks, 2008 for a full discussion) that mindfulness practice may very well facilitate the development of this more economical mode of using and allocating cognitive (in particular) attentional resources. It should be noted that the automated attentional processes found in those experienced in mindfulness practice is in marked contrast to the control-based approaches most typically found in traditional PST approaches, in which regular and ongoing cognitive activity is inherently required to first notice and then correct internal experiences seen as problematic. In addition, Kee and Wang (2008) found that higher levels of dispositional flow and greater self-reported use of mental skills were associated with higher levels of mindfulness. Similarly, Bernier and colleagues have found a strong relationship between mindfulness and flow (2009). These two latter findings are consistent with theoretical connections made between mindfulness and flow as suggested by Gardner and Moore in their 2004 initial article on the Mindfulness-Acceptance-Commitment approach to performance enhancement. Finally, a recent study by Gooding and Gardner (2009) evaluated the relationship between mindfulness, trait arousal, preshot routine, and basketball free throw shooting percentage. Findings showed that free throw skill, 
basketball experience, and levels of mindfulness each predicted game free throw percentage, while trait arousal and consistency and/or length of preshot routine did not. In fact, the data from this study indicated that a one standard deviation increase in mindfulness score would result in a $5.75 \%$ increase in competitive free throw percentage, certainly suggesting the utility of an intervention strategy designed to enhance mindfulness and experiential acceptance.

\section{Intervention Studies Within Clinical/Counseling Psychology}

During the last decade, there has been nothing short of an explosion in clinical studies evaluating the efficacy of acceptance-based behavioral therapies. While a complete review of this literature base is well beyond the scope of this paper (see Roemer \& Orsillo, 2009 for a comprehensive review), it should be noted that numerous studies have demonstrated the efficacy of ABBT for a wide variety of clinical concerns, including substance abuse (e.g., Gifford et al., 2004; Hayes et al., 2004), eating disorders (e.g., Safer, Telch, \& Agras, 2001; Telch, Agras, \& Linehan, 2001), borderline personality disorder (e.g., Gratz \& Gunderson, 2006; Linehan et al., 2007), couples distress (e.g., Christensen, Atkins, Yi, Baucom, \& George, 2006; Jacobson, Christensen, Prince, Cordova, \& Eldridge, 2000), anxiety (e.g., Forman, Herbert, Moitra, Yeomans, \& Geller, 2007) and chronic depression (Ma $\&$ Teasdale, 2004). The plethora of empirical findings has not only suggested that these approaches are efficacious as psychological treatments, but as important, has found that the (acceptance-based) mechanism of change is substantially different than those assumed to exist in traditional (control-based) cognitive behavioral interventions (Forman et al., 2007; Zettle \& Raines, 1989).

\section{Intervention Studies Within Sport Psychology}

Since its inception in 2001, a number of studies (case studies, open trial, and RCT) have demonstrated the efficacy of MAC and closely related interventions for the enhancement of athletic performance and overall well-being. These studies have included a series of case studies (Gardner \& Moore, 2004, 2007; Schwanhausser, 2009) with high-level competitive athletes that suggested enhancements in both process measures of awareness and attention, and outcome measures of competitive performance. In addition, Wolanin (2005) conducted an open trial of MAC, which demonstrated an increase in self and coach ratings of performance, as well as self and coach ratings of attention and practice intensity as compared to treatment controls. Finally, Lutkenhouse conducted a randomized controlled trial in which MAC participants experienced significantly greater increases in measures of attention and flow, as well as coach ratings of performance, than participants receiving traditional PST procedures (Gardner \& Moore, 2007; Lutkenhouse, Gardner, \& Moore, 2007). When viewed in aggregate, these studies suggest the efficacy of MAC for the enhancement of competitive athletic performance.

In addition, a number of empirical studies have used other ABBT interventions that are closely related to MAC both theoretically and procedurally. These studies have been described by the authors as sport-modified versions of Acceptance and Commitment Therapy (ACT), an ABBT most commonly used as a psychological treatment for a vast array of clinical conditions (Hayes, Strosahl, \& Wilson, 1999). 
In a recent study using an adapted version of ACT with elite canoeists, Garcia and colleagues (Garcia, Villa, Cepeda, Cueto, \& Montes, 2004) found that the sportmodified ACT intervention resulted in greater performance on a canoeing training apparatus than a matched control of subjects receiving a hypnosis intervention. In another study, Bernier and colleagues (Bernier, Thienot, Codron, \& Fournier, 2009) found that an acceptance-based intervention adapted for use with elite golfers at a national training institute, resulted in improved golf performance as defined by increases in national rank, when compared with a control condition receiving a traditional PST intervention based on "change" principles.

While additional studies are required that involve multiple sports, use multiple measures of performance across multiple sites, and examine the mechanisms by which performance changes occur, it can readily be said that the evolving body of professional literature clearly suggests that empirical support is fast emerging for the use of MAC and other ABBTs for the purpose of athletic performance enhancement. As such, it is hoped that sport psychology practitioners seeking to adopt an evidence-based approach to practice could comfortably include MAC and other ABBT interventions into his or her professional armamentarium (of course, as long as they do not include change-based approaches as well with the same client, as these orthogonal positions would lead to poor results; Gardner \& Moore, 2007). That being said, MAC has undergone a few modifications and adaptations since its inception. Thus, the remainder of this paper will clarify these changes and suggest some future directions for both practice and research.

\section{Modifications and Enhancements to MAC}

As practitioners and scientists committed to the on-going evolution of psychological interventions based upon the ever expanding scientific foundation of the field, in the years since the development of MAC, we have made a number of evidence-based enhancements to the protocol. These enhancements have been derived from both empirical studies with MAC and within the broad scientific study of ABBT, and of course, through our own experience using MAC on a day-to-day basis. The first published versions of the MAC protocol (Gardner \& Moore, 2004, 2006) suggested that it be delivered in a structured 8-session format, which was broken down into five distinct intervention phases:

1. Psychoeducation Phase

2. Mindfulness Phase

3. Values Identification and Commitment Phase

4. Acceptance Phase

5. Integration and Practice Phase

Any intervention that never evolves is probably a poor one. So, in response to empirical data suggesting that the MAC protocol demonstrated different outcomes based on the absence or presence of subclinical psychological barriers among the clients for whom it was delivered, we decided to make some modifications to the program. It appeared that the necessary adaptations to the protocol needed to address subclinical (i.e., subthreshold DSM-IV-TR) psychological barriers to performance such as worry, perfectionism, transitional, intra/interpersonal issues, etc. (Wolanin, 
2005). The results of our studies, others' research, and our own experience using MAC suggested to us that we should enhance the flexibility in the protocol to allow the practitioner to allot additional time (and attention) on some particularly salient facets of the program (i.e., values-driven behavior vs. emotion-driven behavior, experiential acceptance, mindfulness) with those individuals whose personal issues suggest the need for greater attention to these psychological variables. It should be pointed out that MAC, as is the case with ABBT interventions in general, targets psychological processes that mediate the more obvious problem-outcome connections. For example, empirical evidence has suggested that negative outcomes, both in terms of overall functioning and subjective distress that come from variables assumed to be the primary problem (such as perfectionism and worry), are in fact the result of emotion regulation difficulties in the form of lack of emotional awareness, clarity, acceptance, and distress tolerance (Norman, Oltarzewski, Gardner, \& Moore, 2009; Oltarzewski, Norman, Cambria, Moore, \& Gardner, 2009; Roemer, Lee, Salters-Pedneault, Erisman, Orsillo, \& Mennin, 2009; Santanello \& Gardner, 2007; Wright, Marks, Saxton, Johnson, Cardaciotto, \& Block-Lerner, 2009). In essence, constructs such as perfectionism and worry function as a form of ineffective effort at emotion regulation, and in turn negative subjective and behavioral outcomes ensue. As such, an appropriate evidence-based intervention would not directly target worry or perfectionism, but rather would target the deficit processes that give rise to the maladaptive utilization of worry and perfectionism. Thus, the goal of interventions and (when effective) the mechanism of action for ABBT interventions such as MAC, would be increased emotional awareness, clarity, acceptance, and tolerance of distress. Seen from this perspective, to be effective for clients across the wide range of presenting problems seen by the sport psychologist, utilization of the MAC protocol requires the flexibility to expend appropriate time on these problematic processes without having to abandon the basic structured protocol that has already accumulated evidence for its efficacy. From this evidence-based approach to intervention development, it was determined that to provide the practitioner with the greatest amount of flexibility while still utilizing the structured approach to intervention inherent in the MAC program, the protocol needed to be modified from a fixed 8-session protocol to a flexible 7-module protocol (Gardner \& Moore, 2007). This modification allows the practitioner to flexibly and adequately address specific client needs by expanding the number of sessions available for any module before moving on to the next module in the sequence. Further, in the tradition of science informing practice and in turn practice informing science, our clinical experience using MAC suggested the need for some subtle changes in the sequencing of skills training within the protocol. Specifically, we altered the sequence of skill development in the MAC by introducing the concept of experiential acceptance before the concept of commitment. This subtle change came from our clinical experience that this altered sequence allows for greater client understanding and skill development. The revised protocol maintains the general organization and structure of the original. Yet, it provides a greater capacity to adjust to the needs of those clients who have subclinical issues requiring attention (thus likely taking a few more sessions to complete the seven modules) or those who simply need additional time to understand and effectively integrate the concepts. Yet, of course, the seven modules can still be completed in seven brief sessions for those clients who do not have psychological barriers in need of attention or for 
those who quickly integrate the concepts. Thus, the manualized, step-by-step text published in 2007 on the MAC program detailed the modification to a 7-module approach and discussed the revised MAC protocol in detail. The program's seven modules include the following:

1. Preparing the Client with Psychoeducation

2. Introducing Mindfulness and Cognitive Defusion

3. Introducing Values and Values-Driven Behavior

4. Introducing Acceptance

5. Enhancing Commitment

6. Skill Consolidation and Poise-Combining Mindfulness, Acceptance, and Commitment

7. Maintaining and Enhancing Mindfulness, Acceptance, and Commitment

The other recent enhancement made to the MAC protocol is related to the population served. The original variant of the MAC was directed toward a late adolescent (i.e., college) through older adult population. More recently, there have been developments in modifying the language and presentation of the MAC for use with an early to midadolescent population, with preliminary results suggesting that the protocol can be quite effective with this age group (Schwanhausser, 2009). The adaptations made have not altered the sequencing or intervention targets inherent in the protocol, but have simply adapted the manner in which the protocol is presented to be more understandable and "user-friendly" for this developmentally different population. Given the early success with an adolescent population, it is anticipated that the next step in its development will attempt to use MAC with younger children. In addition, a recent utilization (Gardner \& Moore, 2007) of the MAC program with a group (team) allowed some very interesting application considerations to emerge, which will be further explored in the near future.

\section{Future Directions}

As I stated in a previous paper, "critical thinking requires independence and open skepticism. That is, being neither gullible nor cynical, neither looking for reasons to believe nor reasons to deny" (Moore, 2007, p. 10). As such, as a strong proponent of sound science informing practice, I suggest that while the growing empirical support for MAC and related ABBTs is heartening, there are still advances to be made and ultimately, further modifications to be applied. So, when viewing the developments of the MAC program and related ABBTs applied to sport, and considering the empirical findings suggesting that such approaches are efficacious for use with a wide variety of performance issues, it will be interesting to see where new developments and additional emerging data will take us in the years ahead.

Two newer lines of MAC research with interesting potential include initial efforts to use the MAC protocol in both business and military training applications. In this regard, a case study was recently published on the utilization of the MAC program with a senior corporate executive who needed assistance in maintaining optimal performance states in his respective high-level occupation (Gardner \& Moore, 2007). We have long posited that MAC should be useful across a broad range of performance situations, including sport, corporate, military, performing 
arts, and other high-performing contexts. It is likely that in the course of these expanded uses, additional context-specific modifications and enhancements will be important, while maintaining the appropriate content, sequencing, delivery, and overall integrity of the general MAC program.

In addition to expanding the scope of MAC use, an important next step in the research and development of MAC would be mediational and component analyses; that is, a series of studies that seek to answer the question, which modules (i.e., components) of MAC are essential to its efficacy (in what sequence and by what mechanism of change) and which are superfluous (see Gardner, 2009)? Component analyses would allow for more efficient protocols while maintaining the same efficacy. In addition, it may be that component analyses would ultimately demonstrate that different component sequencing is appropriate for different client problem/issue presentations, and as such would allow for a true evidence-based individualization of the MAC protocol.

\section{Conclusions}

During the past 8 years since the Mindfulness-Acceptance-Commitment (MAC) approach to performance enhancement was first presented at a professional conference, and 5 years since the first iteration of the MAC was published, it can be concluded that the MAC program has accumulated a continually growing empirical base for both its underlying theory and intervention efficacy as a performance enhancement intervention. The MAC protocol has adapted to the ever-changing evidence base in professional psychology to establish itself as a model intervention in sport psychology. MAC research emphasizes not only performance outcomes, but also its mechanisms of action, an area of research that has been nearly totally absent within sport psychology intervention research. While we hope to see additional studies on MAC processes and outcomes to add to the evolution of this approach, according to evidence-based practice standards, the correct, manualized application of MAC can at this time be considered a first line option for performance enhancement efforts with high-level competitive athletes.

\section{References}

Baker, T. B., McFall, R. M., \& Shoham, V. (2009). Current status and future prospects of clinical psychology: Toward a scientifically principled approach to mental and behavioral health. Psychological Science in the Public Interest. 9, 4-144.

Bernier, M., Thienot, E., Codron, R., \& Fournier, J.F. (2009). A multi-study investigation examining the relationship between mindfulness and acceptance approaches and sport performance. Journal of Clinical Sports Psychology, 3, 320-333.

Brefczynski-Lewis, J.A., Lutz, A., Schaefer, H.S., Levinson, D.B., \& Davidson, R.J. (2007). Neural correlates of attentional expertise in long-term meditation practitioners. Proceedings of the National Academy of Sciences of the United States of America, 104, $11483-11488$.

Burton, D. (1989). Winning isn't everything: Examining the impact of performance goals on collegiate swimmers' cognitions and performance. The Sport Psychologist, 3, 105-132.

Chambers, R., Chuen Yee Lo, B., \& Allen, N.B. (2008). The impact of intensive mindfulness training on attentional control, cognitive style and affect. Cognitive Therapy and Research, 32, 303-322. 
Christensen, A., Atkins, D.C., Yi, J., Baucom, D.H., \& George, W.H. (2006). Couple and individual adjustment for two years following a randomized clinical trial comparing traditional vs. integrative behavioral couple therapy. Journal of Consulting and Clinical Psychology, 74, 1180-1191.

Davidson, R.J., Kabat-Zinn, J., Schumacher, J., Rosenkranz, M., Muller, D., Santorelli, S., et al. (2003). Alterations in brain and immune function produced by mindfulness meditation. Psychosomatic Medicine, 65, 564-570.

Daw, J., \& Burton, D. (1994). Evaluation of a comprehensive psychological skills training program for collegiate tennis players. The Sport Psychologist, 8, 37-57.

Forman, E.M., Herbert, J.D., Moitra, E., Yeomans, P.D., \& Geller, P.A. (2007). A randomized controlled effectiveness trial of acceptance and commitment therapy and cognitive therapy for anxiety and depression. Behavior Modification, 31, 772-799.

Garcia, R.F., Villa, R.S., Cepeda, N.T., Cueto, E.G., \& Montes, J.M.G. (2004). Efecto de la hypnosis y la terapia de aceptcion y compromiso (ACT) en la mejora de la fuerza fisica en piraguistas. International Journal of Clinical and Health Psychology, 4, 481-493.

Gardner, F.L. (2001). Principles and practices of acceptance-based sport psychology. Grand rounds lecture presented at the Department of Psychiatry Grand Rounds at North Shore University Medical Center and Health System, Manhasset, NY.

Gardner, F.L. (2009). Efficacy, mechanisms of change, and the scientific development of sport psychology. Journal of Clinical Sports Psychology, 3, 139-155.

Gardner, F.L., \& Moore, Z.E. (2004). A Mindfulness-Acceptance-Commitment (MAC) based approach to athletic performance enhancement: Theoretical considerations. Behavior Therapy, 35, 707-723.

Gardner, F.L., \& Moore, Z.E. (2006). Clinical sport psychology. Champaign, IL: Human Kinetics.

Gardner, F.L., \& Moore, Z.E. (2007). The psychology of enhancing human performance: The Mindfulness-Acceptance-Commitment (MAC) approach. New York: Springer Publishing.

Gifford, E.V., Kohlenberg, B.S., Hayes, S.C., Antonuccio, D.O., Piasecki, M.M., RasmussenHall, M.L., et al. (2004). Acceptance-based treatment for smoking cessation. Behavior Therapy, 35, 689-705.

Gooding, A., \& Gardner, F.L. (2009). An empirical investigation on the relationship between mindfulness, pre shot routine, and basketball free throw percentage. Journal of Clinical Sports Psychology, 3, 303-319.

Gratz, K.L., \& Gunderson, J.G. (2006). Preliminary data on an acceptance-based emotion regulation group intervention for deliberate self-harm among women with borderline personality disorder. Behavior Therapy, 37, 25-35.

Hanin, Y.L. (1980). A study of anxiety in sport. In W.F. Straub (Ed.), Sport psychology: An analysis of athlete behavior (pp. 236-249). Ithaca, NY: Movement Publications.

Hardy, L., Jones, G., \& Gould, D. (1996). Understanding psychological preparation for sport: Theory and practice of elite performers. New York: Wiley.

Hayes, S.C., Bissett, R., Roget, N., Padilla, M., Kohlenberg, B.S., Fisher, G., et al. (2004). The impact of acceptance and commitment training and multicultural training on the stigmatizing attitudes and professional burnout of substance abuse counselors. Behavior Therapy, 35, 821-835.

Hayes, S.C., Strosahl, K., \& Wilson, K.G. (1999). Acceptance and Commitment Therapy: An experiential approach to behavior change. New York: Guilford Press.

Jacobson, N.S., Christensen, A., Prince, S.E., Cordova, J., \& Eldridge, K. (2000). Integrative couple therapy: An acceptance-based, promising new treatment for couple discord. Journal of Consulting and Clinical Psychology, 68, 351-355.

Jha, A.P., Krompinger, J., \& Baime, M.J. (2007). Mindfulness training modifies subsystems of attention. Cognitive, Affective \& Behavioral Neuroscience, 7, 109-119. 
Kabat-Zinn, J. (2005). Coming to our senses: Healing ourselves and the world through mindfulness. New York: Hyperion.

Kee, Y.H., \& Wang, C.K.J. (2008). Relationships between mindfulness, flow dispositions, and mental skills adoption: A cluster analytic approach. Psychology of Sport and Exercise, 9, 393-411.

Linehan, M.M., Comtois, K.A., Murray, A.M., Brown, M.Z., Gallop, R.J., Heard, H.L., et al. (2007). Two-year randomized controlled trial and follow-up of dialectical behavior therapy vs. therapy by experts for suicidal behaviors and borderline personality disorder. Archives of General Psychiatry, 63, 757-766.

Lutkenhouse, J., Gardner, F.L., \& Moore, Z.E. (2007). A randomized controlled trial comparing the performance enhancement effects of Mindfulness-Acceptance-Commitment $(M A C)$ performance enhancement and psychological skills training procedures. Manuscript in preparation.

Ma, S.H., \& Teasdale, J.D. (2004). Mindfulness-based cognitive therapy for depression: Replication and exploration of differential relapse prevention effects. Journal of Consulting and Clinical Psychology, 72, 31-40.

Marks, D.R. (2008). The Buddha's extra scoop: Neural correlates of mindfulness practice and their relevance for clinical sport psychology. Journal of Clinical Sports Psychology, 2, 216-241.

Maynard, I.W., Smith, M.J., \& Warwick-Evans, L. (1995). The effects of a cognitive intervention strategy on competitive state anxiety and performance in semi-professional soccer players. Journal of Sport \& Exercise Psychology, 17, 428-446.

Moore, Z.E. (2003). Toward the development of an evidence based practice of sport psychology: A structured qualitative study of performance enhancement interventions (Doctoral Dissertation, La Salle University, 2003). Dissertation Abstracts International-B, 64 (10), 5227. (UMI No. 3108295).

Moore, Z.E. (2007). Critical thinking and the evidence-based practice of sport psychology. Journal of Clinical Sports Psychology, 1, 9-22.

Moore, Z.E., \& Gardner, F.L. (2001, October). Taking applied sport psychology from research to practice: Integrating empirically supported interventions into a self-regulatory model of athletic performance. Workshop presented at the meeting of the Annual Conference of the Association for the Advancement of Applied Sport Psychology, Orlando, FL.

Moore, Z.E., \& Gardner, F.L. (2002, August). Psychological skills training for athletic performance enhancement: An evidence-based approach. Paper presented at the Annual Convention of the American Psychological Association, Chicago, Illinois.

Moore, Z.E., \& Gardner, F.L. (2003, August). A protocol for Mindfulness-Acceptance Commitment (MAC) approach to athletic performance enhancement. Paper presented at the Annual Conference of the American Psychological Association, Toronto, Canada.

Murphy, S.M., \& Woolfolk, R. (1987). The effects of cognitive interventions on competitive anxiety and performance on a fine motor skill accuracy task. International Journal of Sport Psychology, 18, 152-166.

Norman, J., Oltarzewski, J., Gardner, F.L., \& Moore, Z.E. (2009, November). The impact of gender on the relationship between experiential avoidance, emotional clarity, and bulimic behavior. Paper presented at the Annual Conference of the Association for Behavioral and Cognitive Therapies, New York, NY.

Oltarzewski, J., Norman, J., Cambria, N., Moore, Z.E., \& Gardner, F.L. (2009, November). A comparison of perfectionism among eating disordered and non-eating disordered individuals. Paper presented at the Annual Conference of the Association for Behavioral and Cognitive Therapies, New York, NY.

Roemer, L., Lee, J.K., Salters-Pedneault, K., Erisman, S.M., Orsillo, S.M., \& Mennin, D.S. (2009). Mindfulness and emotion regulation difficulties in generalized anxiety disorder: Preliminary evidence for independent and overlapping contributions. Behavior Therapy, 40, 142-154. 
Roemer, L., \& Orsillo, S.M. (2009). Mindfulness \& acceptance-based behavioral therapies in practice. New York: Guilford Press.

Safer, D.L., Telch, C.F., \& Agras, W.S. (2001). Dialectical behavior therapy for bulimia nervosa. The American Journal of Psychiatry, 158, 632-634.

Santanello, A.W., \& Gardner, F.L. (2007). The role of experiential avoidance in the relationship between maladaptive perfectionism and worry. Cognitive Therapy and Research, 31, 319-332.

Sbrocco, T., \& Barlow, D.H. (1996). Conceptualizing the cognitive component of sexual arousal: Implications for sexuality research and treatment. In P.M. Salkovskis (Ed.), Frontiers of cognitive therapy (pp. 419-449). New York: Guilford Press.

Schwanhausser, L. (2009). Application of the Mindfulness-Acceptance-Commitment (MAC) Protocol with an Adolescent Springboard Diver: The Case of Steve. Journal of Clinical Sports Psychology, 3, 377-396.

Telch, C.F., Agras, W.S., \& Linehan, M.M. (2001). Dialectical behavior therapy for binge eating disorder. Journal of Consulting and Clinical Psychology, 69, 615-623.

Wegner, D.M., Ansfield, M., \& Pilloff, D. (1998). The putt and the pendelum: Ironic e effects of the mental control of action. Psychological Science, 9, 196-199.

Wegner, D.M., \& Zanakos, S. (1994). Chronic thought suppression. Journal of Personality, 62, 615-640.

Weinberg, R., Seabourne, T.G., \& Jackson, A. (1981). Effects of visuo-motor behavior rehearsal, relaxation, and imagery on karate performance. Journal of Sport Psychology, 3, 228-238.

Wolanin, A.T. (2005). Mindfulness-Acceptance-Commitment (MAC) based performance enhancement for Division I collegiate athletes; A preliminary investigation (Doctoral Dissertation, La Salle University, 2003). Dissertation Abstracts International-B, 65 (7), 3735-3794.

Wolanin, A., Gardner, F., \& Moore, Z. (2003, August). A preliminary investigation of Mindfulness-Acceptance Commitment (MAC) based performance enhancement. Paper presented at the Annual Conference of the American Psychological Association, Toronto, Canada.

Wright, T., Marks, D.R., Saxton, V., Johnson, A., Cardaciotto, L., \& Block-Lerner, J. (2009). Does distress tolerance account for the relationship between dispositional mindfulness and psychological distress. Paper presented at the Annual Conference of the Association for Behavioral and Cognitive Therapies, New York, NY.

Zettle, R.D., \& Raines, J.C. (1989). Group cognitive and contextual therapies in treatment of depression. Journal of Clinical Psychology, 45, 436-445. 